\title{
Neuroprotectin D1 upregulates Iduna expression and provides protection in cellular uncompensated oxidative stress and in experimental ischemic stroke
}

\author{
Ludmila Belayev $^{1,3}$, Pranab K Mukherjee ${ }^{1,3}$, Veronica Balaszczuk ${ }^{1,3}$, Jorgelina M Calandria ${ }^{1,3}$, Andre Obenaus ${ }^{2}$, Larissa Khoutorova ${ }^{1}$, \\ Sung-Ha Hong ${ }^{1}$ and Nicolas G Bazan ${ }^{* 1}$
}

Ring finger protein 146 (Iduna) facilitates DNA repair and protects against cell death induced by NMDA receptor-mediated glutamate excitotoxicity or by cerebral ischemia. Neuroprotectin D1 (NPD1), a docosahexaenoic acid (DHA)-derived lipid mediator, promotes cell survival under uncompensated oxidative stress (UOS). Our data demonstrate that NPD1 potently upregulates Iduna expression and provides remarkable cell protection against UOS. Iduna, which was increased by the lipid mediator, requires the presence of the poly(ADP-ribose) (PAR) sites. Moreover, astrocytes and neurons in the penumbra display an enhanced abundance of Iduna, followed by remarkable neurological protection when DHA, a precursor of NPD1, is systemically administered $1 \mathrm{~h}$ after $2 \mathrm{~h}$ of ischemic stroke. These findings provide a conceptual advancement for survival of neural cells undergoing challenges to homeostasis because a lipid mediator, made 'on demand,' modulates the abundance of a critically important protein for cell survival.

Cell Death and Differentiation (2017) 24, 1091-1099; doi:10.1038/cdd.2017.55; published online 21 April 2017

Neuronal cell death involves uncompensated oxidative stress (UOS) as an early event. Parthanatos, a form of cell death, is dependent of poly(ADP-ribose) polymerase-1 (PARP-1). ${ }^{1-4}$ PARPs catalyze the transfer of ADP-ribose from nicotinamide adenine dinucleotide (NAD) to target proteins and are fundamental for genomic integrity, the cell cycle and gene expression. ${ }^{5,6}$ Suppression of PARP expression reduces stroke volume. ${ }^{7,8}$ Iduna (ring finger protein 146 (RNF146) is a PARsylation-directed ring finger E3 ubiquitin ligase that plays a key role in protein quality control, fosters DNA repair and provides protection against parthanatos in cerebral ischemia. ${ }^{2,7,9}$ Since it is unknown what key cellular-response signals of neural homeostatic disruptions are linked to Iduna expression, we asked if the lipid mediator neuroprotectin D1 (NPD1) could function in this capacity. NPD1 is derived from the omega-3 docosahexaenoic acid (DHA), which is enriched in the nervous system. ${ }^{10}$ Neuroectoderm-derived post-mitotic retinal pigment epithelial (RPE) cells are protected by NPD1 when exposed to UOS. ${ }^{11}$ Also, during early reperfusion after cerebral ischemia NPD1 synthesis increases in the brain. When NPD1 is administered under these conditions, downregulation of leukocyte infiltration, attenuation of proinflammatory signaling and decreased infarct size takes place. ${ }^{12,13}$ Moreover, systemically administered DHA is used to synthesize NPD1 in the brain after an ischemic stroke. Based on both of these observations in the eye and brain, we decided to explore whether or not NPD1 modulates Iduna expression using RPE cells undergoing UOS ${ }^{14}$ and in an ischemic stroke model. ${ }^{15}$ Our results demonstrate that NPD1 upregulates Iduna expression and provides protection against UOS at the cellular level in a PAR-binding-dependent fashion. Moreover, in ischemic stroke, Iduna expression is downregulated in the penumbra and is associated with neurological deficits. DHA, as a precursor of NPD1, counteracts these deficits by increasing Iduna expression in neurons and astrocytes after stroke onset. Thus our findings establish a causal relationship whereby NPD1 modulates Iduna abundance and cell survival after neural injury/stroke, resulting in neurological protection.

\section{Results}

NPD1 upregulates Iduna abundance in RPE cells undergoing UOS. We used two different types of human RPE cells in separate cultures: (1) a spontaneously transformed cell, ARPE-19, and (2) primary RPE cells. NPD1 enhanced expression of Iduna in both cell types. The effect of NPD1 on Iduna expression peaked at $6 \mathrm{~h}$ after the onset of UOS (Figure 1b). A dose-dependent curve showed an increase of Iduna expression starting at $25 \mathrm{nM}$ NPD1 in both cell types (Figures 1c and d). NPD1 and UOS alone did not enhance expression of Iduna (Figures $1 b-d, 2 a$ and $3 b-h$ ). NPD1 bioactivity was specific since other protective lipid messengers (lipoxin A4 and 15-epi-LPX) were unable to enhance Iduna expression in the RPE cells undergoing UOS (Figure 1e). Altogether these results suggest that NPD1

\footnotetext{
${ }^{1}$ Neuroscience Center of Excellence, School of Medicine, Louisiana State University Health Sciences Center, New Orleans, LA, USA and ${ }^{2}$ Department of Pediatrics, Loma Linda University, Loma Linda, CA, USA

*Corresponding author: NG Bazan, Neuroscience Center of Excellence, School of Medicine, Louisiana State University Health Sciences Center, 2020 Gravier St, New Orleans, LA 70112, USA. Tel: +504 599 0831; Fax: +504 568 5801; E-mail: nbazan@|suhsc.edu

${ }^{3}$ These authors contributed equally to this work.

Received 16.8.16; revised 17.3.17; accepted 21.3.17; Edited by RA Knight; published online 21.4.2017
} 
a

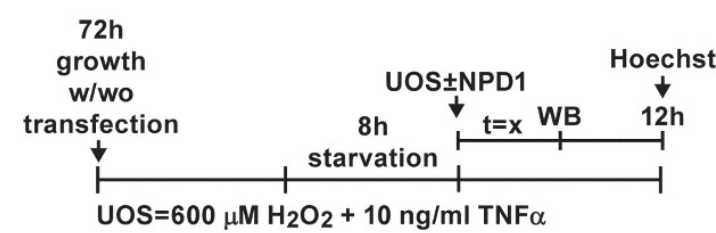

b
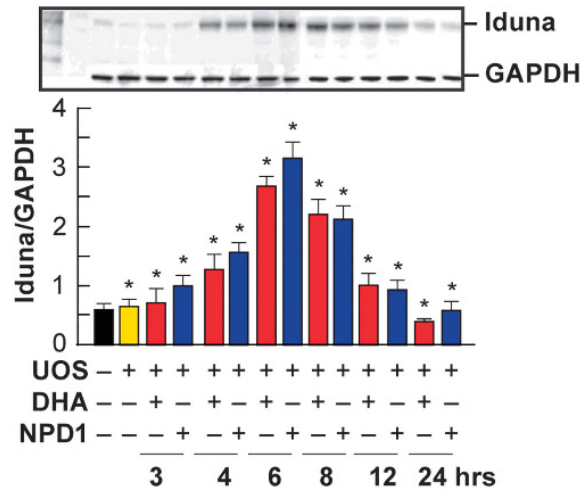

c

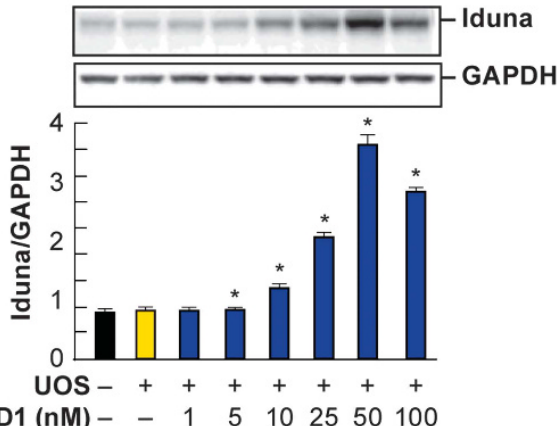

d
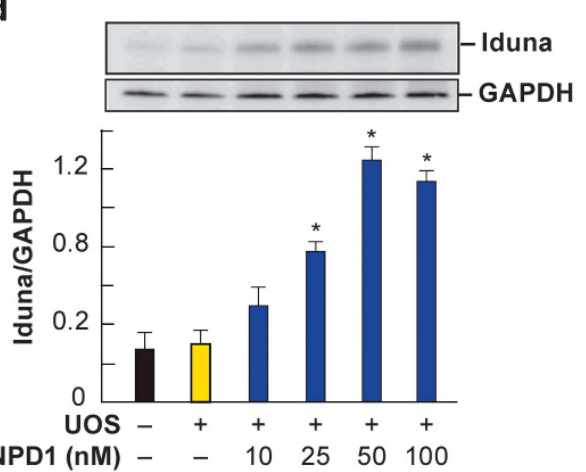

e

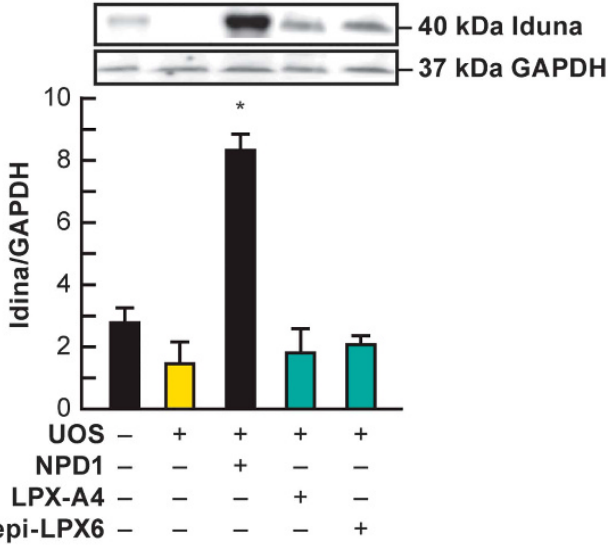

f
Non-transfected cells

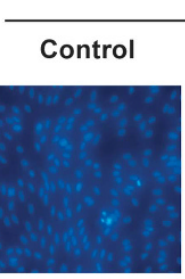

Hoechst Stain
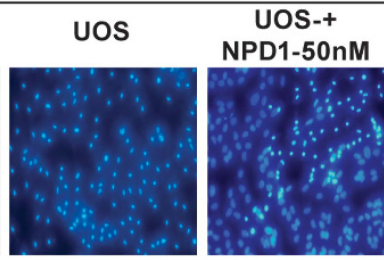

NPD1-50nM
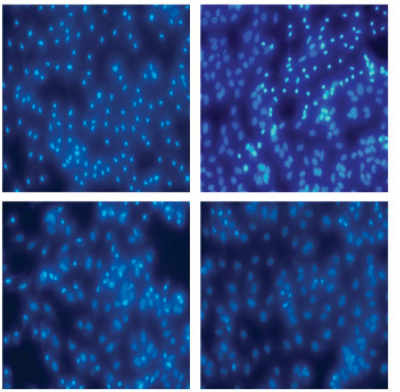

Iduna cfugwRNF146 GFP-(Human) transfected
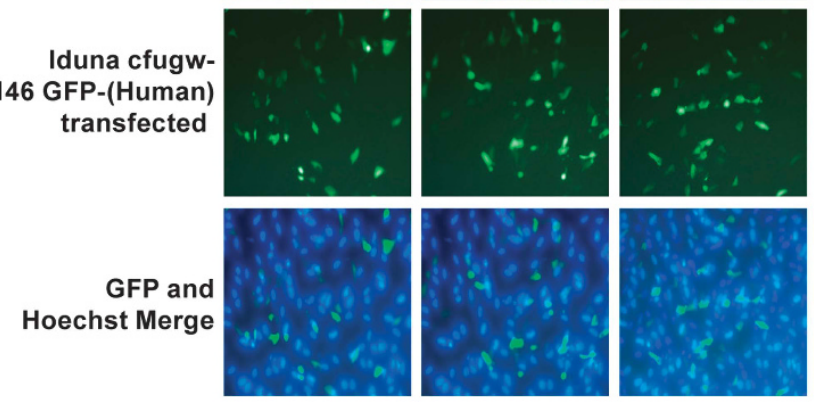

g

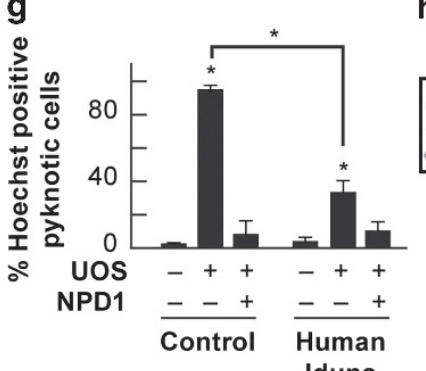

h

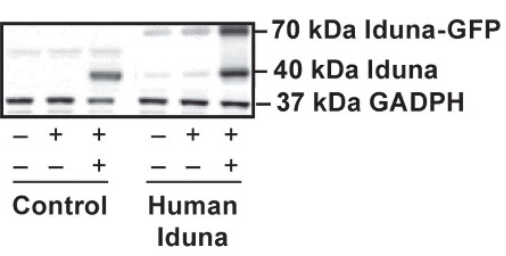

Figure 1 NPD1 upregulates Iduna expression in RPE cells undergoing UOS. (a) Experimental design timeline. Cells were transfected and recovered for $72 \mathrm{~h}$. After $8 \mathrm{~h}$ of incubation in low serum medium (starvation), UOS was induced with $600 \mu \mathrm{M} \mathrm{H}_{2} \mathrm{O}_{2}$ plus $10 \mathrm{ng} / \mathrm{ml}$ of TNF- $\alpha$ in the presence and absence of NPD1. After a variable period ( $t=x$ ) that ranged between 3 and $24 \mathrm{~h}(\mathbf{b})$ or $6 \mathrm{~h}$ (c-e and $\mathbf{h}$ ), cells were harvested for western blot. For Hoechst staining, cells were fixed after $12 \mathrm{~h}$ of treatment (f and $\mathbf{g}$ ). (b) Western blot of the time course of Iduna expression in ARPE-19 cells. (c and d) NPD1 (1-100 nM)-mediated expression of Iduna in UOS-treated ARPE-19 (c) and hRPE cells (d). (e) Specific expression of Iduna by NPD1, since the lipid mediators lipoxin A4 (LPX-A4) and 15-epi-LPX are inactive in ARPE-19 cells. (f) Representative images of Iduna expression by transient transfection of human Iduna construct (Iduna-146-GFP-Hu), (g) quantification of Hoechst-positive ARPE-19 cells and (h) western blot from transfection with Iduna human constructing ARPE-19 cells undergoing UOS and treated with $50 \mathrm{nM}$ NPD1. Bars represent data averages of three repeats (technical replicas) of three independent experiments (biological replicas). ${ }^{*} P<0.05$ 
a

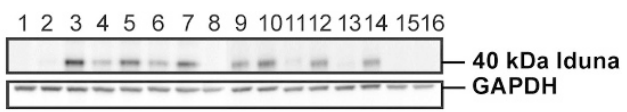

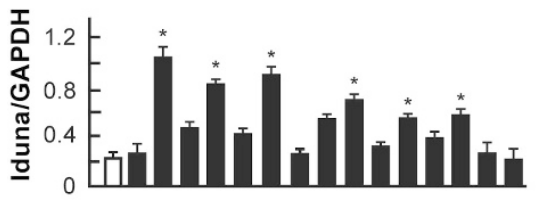

b

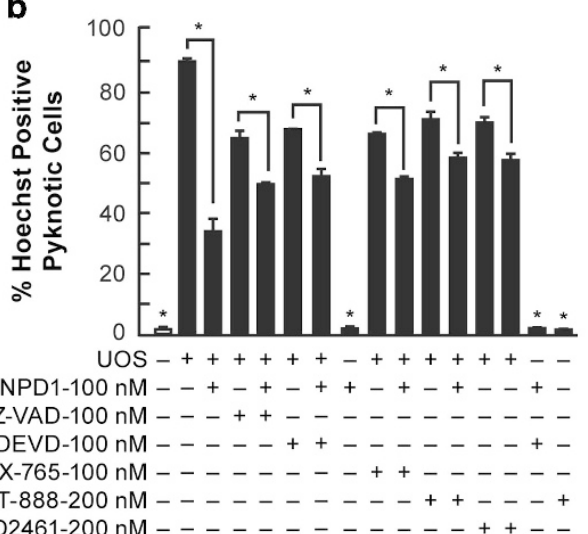

Figure 2 NPD1 enhances survival induced by inhibitors of PARP or caspases. (a) Iduna expression was assessed by western blot (upper panel). Densitometry shows expression of Iduna relative to GAPDH. (b) Hoechst-positive cell quantification of caspase and PARP inhibitors and their effect on ARPE-19 cells undergoing UOS in the presence and absence of NPD1 (lower panel). Bars represent data averages of three repeats (technical replicas) of three independent experiments (biological replicas). ${ }^{*} P<0.05$

selectively induces Iduna upregulation, and both NPD1 and UOS are required for Iduna upregulation.

Iduna overexpression offsets UOS-induced apoptosis in RPE cells. We then tested whether Iduna overexpression would attenuate UOS-stimulated cell death. Overexpression of human Iduna (Iduna-146-GFP-Hu) in RPE cells (Figures $1 \mathrm{f}$ and g) attenuated cell death induced by UOS (Figures 1e and $\mathrm{f}$ ), and the addition of NPD1 (50 nM) to the transfected cells further enhanced inhibition of cell death. Transfection efficiencies of the plasmids were 65 to $70 \%$ for the human Iduna construct (Figures 1f, third row). Moreover, the addition of Caspase-3 and Caspase-1 inhibitors, Z-DEVD and VX-765 , reduced approximately $25 \%$ of the cell death observed in cells undergoing UOS (Figure 2). The addition of $100 \mathrm{nM}$ NPD1 to the caspases inhibitors decreased $43 \%$ the level of cell death (Figure 2b) and increased Iduna expression in each case (Figure 2a). The difference in cell death observed between inhibitors and inhibitors plus NPD1 suggest that there is another mechanism of cell death independent of the caspases. Inhibitors of PARP, ABT-888 and AZD2461 showed trends similar to those observed with the caspase inhibitors (Figure 2b). These results suggest that caspase-dependent and PARP-dependent mechanisms are both part of the cell death triggered by UOS, and Iduna expression is involved in the NPD1-mediated protection of RPE cells.

Next we asked whether or not Iduna was involved in the NPD1-mediated survival effects. For this purpose, we used plasmids carrying shRNA-Iduna to silence the protein or YRAA-Iduna. The shRNA was introduced to silence the expression of Iduna, whereas the YRAA-Iduna has a mutation that prevents Iduna from binding with PAR. ${ }^{2}$ Our results show that NPD1 was unable to protect RPE cells against UOS after transfection/expression of either the shRNA-Iduna or YRAAIduna constructs (Figure 3a). Cells expressing shRNA-Iduna did not express the protein as expected (Figures $3 b$ and $c$ ), and cells transfected with the YRAA-Iduna construct displayed mutant Iduna, which lacks PAR-binding sequences (Figures 3b and c).

Ablation of PAR-binding sites affects NPD1-mediated Iduna increase. Because PAR regulates protein function through binding at the poly(ADP-ribose) (PAR)-binding sites, ${ }^{16}$ we then asked whether the NPD1-mediated Iduna increase was dependent on PAR binding in RPE cells undergoing UOS. We showed that YRAA mutant Iduna expression in RPE cells reduced the amount of wild-type endogenous Iduna protein in comparison with the non-YRAAexpressing cells undergoing UOS plus NPD1 (Figures $3 \mathrm{~b}$ and c). In agreement with the latter observation, overexpression of the wild-type Iduna-GFP open reading frame increased the content of endogenous Iduna (Figure 1h). These results suggest that the presence of non-PAR-binding Iduna induce a decrease in the endogenous counterpart and that an interaction between different types of Iduna occurred to modify the overall turnover of the protein (Figures $3 b$ and $c$ ). The PARsylation-directed ubiquitination mediated by Iduna is analogous to the phosphorylation-directed ubiquitination catalyzed by the Skp1-Cul1-F-box (SCF) E3 ubiquitin complex. $^{9,16}$ Therefore, modulation of the PAR-binding activity of Iduna by NPD1 should play a key role in proteasomal protein degradation and cell protection at early stages of neuro-pathophysiology. We found that Iduna expression did not occur in the control cells or in UOStreated ARPE-19 cells (Figures $3 b-h$ ). In contrast, Iduna expression in ARPE-19 cells under UOS is dependent upon the concentration of NPD1 (Figure 3f). Moreover, the nuclear fraction (but not the cytoplasmic fraction) of ARPE-19 cells displayed an increase in the Iduna protein (Figure $3 \mathrm{~g}$ ). In addition, NPD1 induced nuclear Iduna increase (yellow) only in UOS+NPD1-treated cells (Figure 3h, lower row: green in $2 \mathrm{D}$ images and yellow in 3D and merged images). Cells that were not treated with NPD1 did not display protein translocation (Figure 3h, upper and middle rows), further supporting the mediator role of NPD1 in this process.

DHA, as a precursor of NPD1, counteracts ischemic stroke neurological deficits with concomitant increase of Iduna in neurons and astrocytes in the penumbra. Having established that NPD1 facilitates Iduna expression at the cellular level, we decided to test whether this mediator also elicits a similar function in ischemic stroke because the neuroprotective bioactivity of this protein has been shown previously under similar conditions. We systemically administered DHA (i.v.) $1 \mathrm{~h}$ after the onset of reperfusion following $2 \mathrm{~h}$ of middle cerebral artery (MCA) occlusion (MCAo). It has been shown that NPD1 synthesis from systemically administered DHA takes place in the brain under these conditions. ${ }^{15}$ 


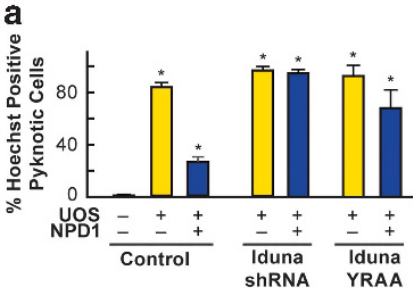

b
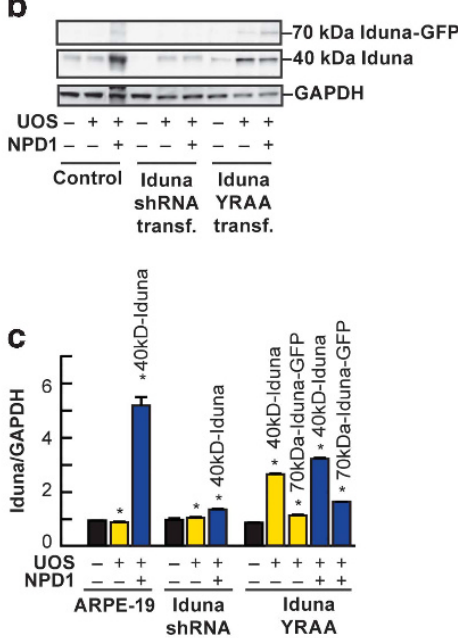

d

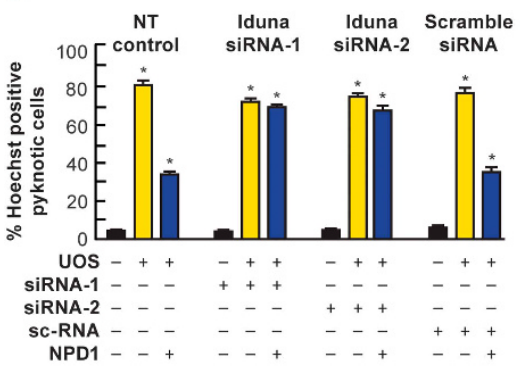

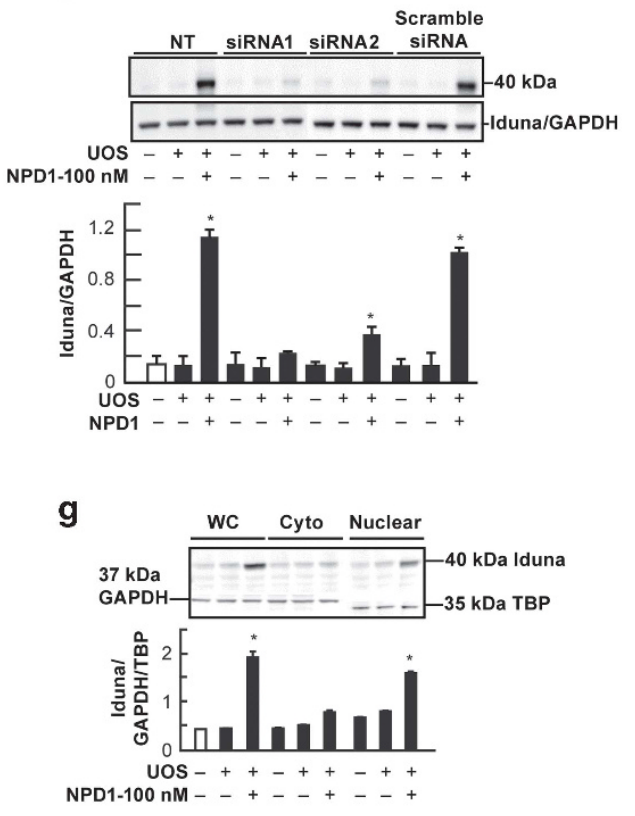

NPD1-100 $\mathrm{nM}-++-++-++$
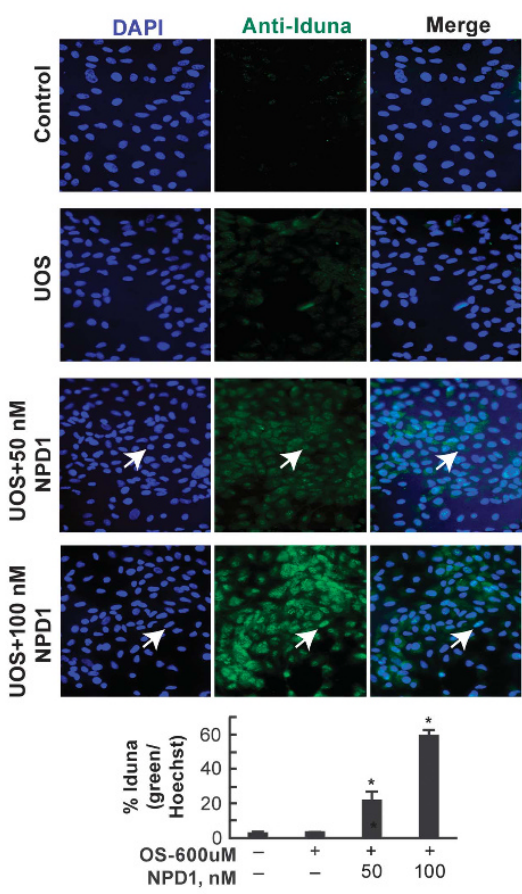

h
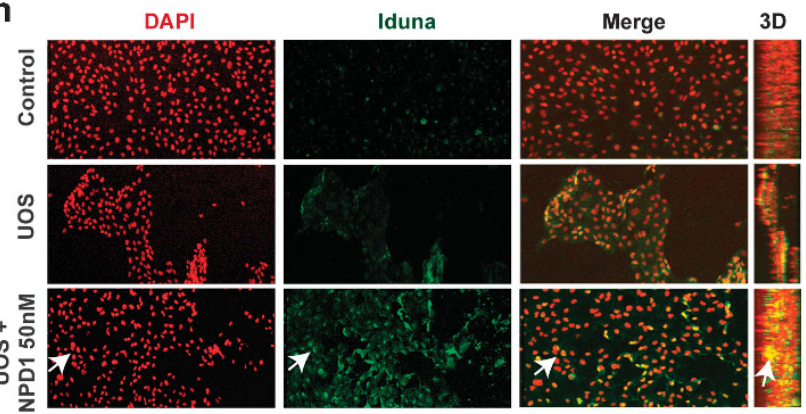

Figure 3 NPD1 mediates expression and translocation of Iduna and PAR dependency. (a) Effect of NPD1 on apoptosis in ARPE-19 cells transfected with either shRNA-Iduna or YRAA-Iduna construct. (b) Western blot and (c) densitometric quantification of Iduna expression by NPD1 in ARPE-19 cells and controls transfected with shRNA-Iduna and YRAA-Iduna construct. (d and e) Silencing of Iduna using two different siRNAs (siRNA1 and siRNA2) and a control nonspecific targeting siRNA (scramble). (d) Hoechst staining of transfected or non-transfected ARPE-19 cells undergoing UOS in the presence or absence of NPD1 and (e) Western blot and densitometry of Iduna expression. (f) Endogenous expression of Iduna in response to NPD1. Representative images of immunostaining of ARPE-19 cells undergoing UOS in the absence or presence of 50 or 100 nM NPD1 and quantification of Iduna-positive cells. ( $\mathbf{f}$ and $\mathbf{h}$ ) Arrows mark examples of the Iduna-positive nuclei. ( $\mathbf{g}$ and $\mathbf{h}$ ) Nuclear translocation of Iduna by NPD1. (g) Western blot analysis and densitometry of whole cell (WC), cytoplasmic (Cyto) and nuclear fractions of ARPE-19 cells undergoing UOS in the presence or absence of $100 \mathrm{nM}$ NPD1. Whole cell and cytoplasmic fractions where standardized using GAPDH and nuclear fraction using TBP. (h) Z-stacks flattened 2D images (red = DAPI first column; green = Iduna, second column; merged channels, third column) and 3D representation of ARPE-19 cells immunocytochemistry targeting endogenous Iduna. Bar $=200 \mu$ m. Arrows indicate co-localization (yellow) of both the DAPI and Iduna signal in the nucleus. Bars represent data averages of three repeats (technical replicas) of three independent experiments (biological replicas). ${ }^{*} P<0.05$

Also, this model of MCAo leads to an improved neurologic score in NPD1-treated rats (Figure 4a). ${ }^{12}$ Our studies show that DHA treatment rescued the ischemic core and penumbra on days 3 and 7 (Figure 4b), decreased edema T2 values (Figures $4 \mathrm{c}$ and d) and reduced cortical, subcortical and total lesion volumes (Figure 4e), as shown in the 3D lesion volumes (Figure 4d). Post-ischemic activation of PARP-1 occurs in neurons, astrocytes, microglial cells, endothelia and infiltrating leukocytes (Figure 5). Our data show that systemically administered DHA after MCAo heightened Iduna abundance in neurons on day 1 (Figures $5 a$ and b) and in astrocytes on days 1, 3 and 7 (Figures $5 a$ and $b$ ). In addition, sham-MCAo rats treated with DHA showed no increase of Iduna content (Figure 5h). This was ascertained by coexpression with neuron-specific nuclear protein (NeuN) and glial fibrillary acidic protein (GFAP) (Figure 5c). Iduna was augmented $70 \%$ in the ipsilesional brain cortex (bregma level +1.2 and $-2.8 \mathrm{~mm}$ ) in DHA-treated animals. Saline-treated animals displayed a $30-40 \%$ increase in Iduna abundance one day after MCAo. The presence of Iduna in the contralateral side was cytoplasmatic, whereas in the ipsilesional side Iduna was nuclear (Figure $5 \mathrm{~d}$ ). DHA elicited Iduna 
a

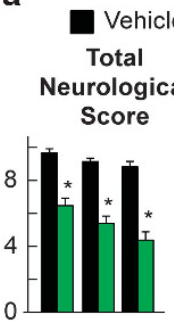

Visual

Placing

Sideways

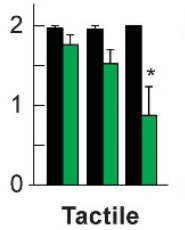

Tactile

Lateral

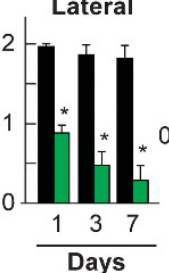

Vehicle $\square$ DHA

Postural Reflex
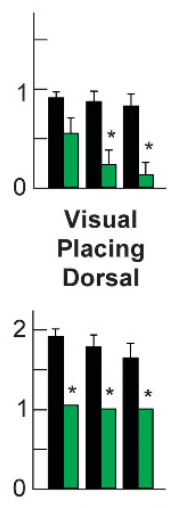

Proprioceptive Placing

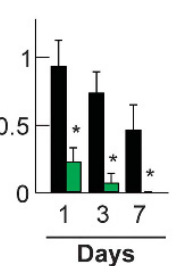

b
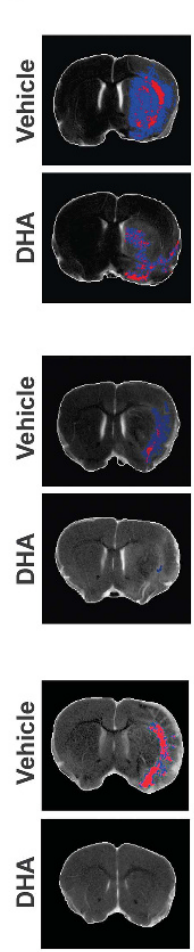

Day 1

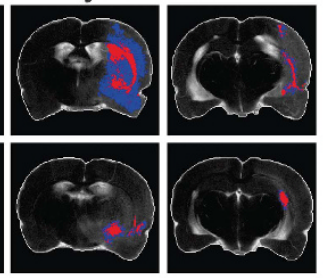

Day 3

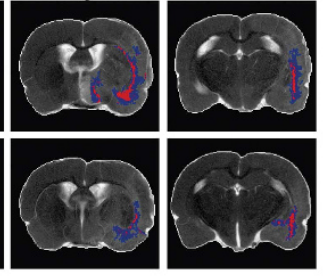

Day 7
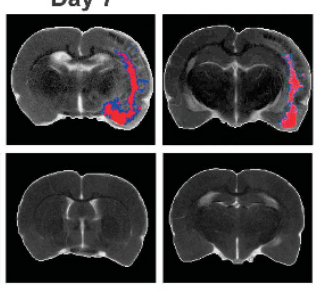

C Total $\square$ Cortex $\square$ Subcortex
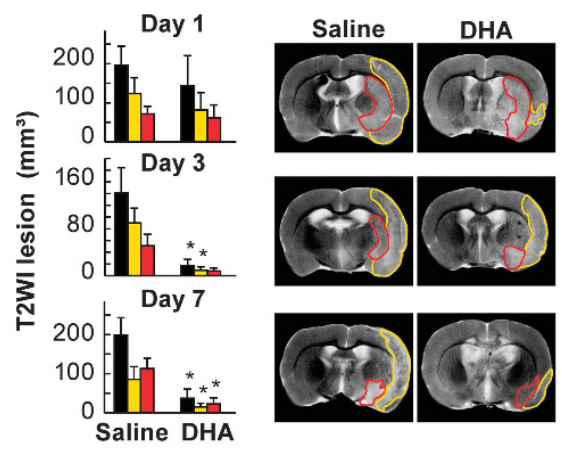

d

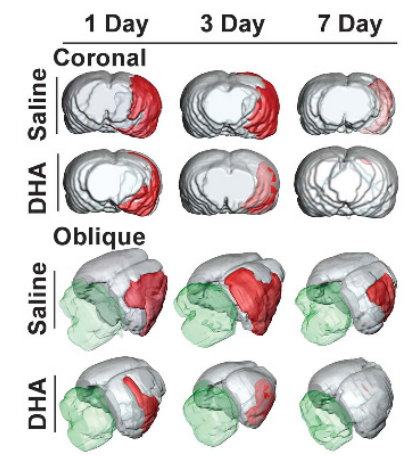

e

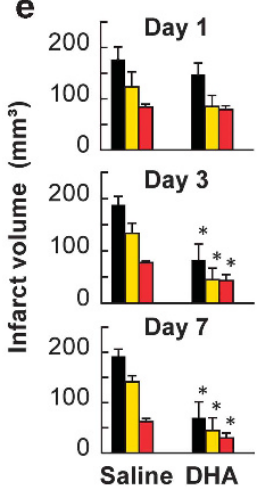

Figure 4 DHA improves neurological scores, protects ischemic penumbra, decreases MRI lesions and diminishes infarct volumes. (a) Time course of neurologic assessment (normal score $=0$, maximal score $=12$ ), postural reflex and visual, tactile and proprioceptive forelimb placing reactions (normal score $=0$, maximal score $=2$ ). DHA $(n=6)$ or vehicle $(n=7)$ were administered $3 \mathrm{~h}$ after the onset of MCAo. DHA-treated rats improved all scores on days 1,3 and 7 . (b) High-resolution ex vivo MRI on days 1,3 and 7 . Core and penumbra were extracted from the entire brain and lesion volumes were determined using MRI. Core (red) and penumbral (blue) tissues were automatically identified in saline- and DHA-treated animals using the computational MRI method Hierarchal Region Splitting for penumbra identification. DHA treatment reduced lesions in the ischemic core and penumbra on days 3 and 7. (c) Cortical, subcortical and total lesion volumes, computed from T2WI and representative T2-weighted images (T2WI) from vehicle- and DHA-treated rats on days 1, 3 and 7. DHA treatment reduced T2 values within the lesion on days 3 and 7 . T2 hyper-intensities were observed in the cortex and striatum of salinetreated rats, consistent with ongoing edema formation. In contrast, DHA-treated rats had only a small cortical and subcortical lesion on day 7. (d) 3D reconstructions of MRIderived lesion volumes from high-resolution T2WI from vehicle- and DHA-treated rats on days 1, 3 and 7 after MCAo. Vehicle-treated rats showed large cortical and subcortical lesion volumes (red color) that slowly decreased over the course of 7 days. By contrast, lesion volume was dramatically reduced in rats treated with DHA and was localized mostly in the subcortical areas. (e) Histopathology on days 1, 3 and 7 after MCAo. Total infarct volume was corrected for brain swelling. DHA treatment dramatically reduced cortical, subcortical and total infarct volumes. Values shown are means \pm S.D. ${ }^{*} P<0.05$, versus saline group (repeated measures analysis of variance followed by Bonferroni tests)

localization in the cytoplasm, nucleus and neuronal projections (Figure 5e), and it increased Iduna abundance in the ipsilesional and contralateral cortex and the subcortex on days 1 and 3, compared with vehicle-treated rats (Figure 5f), with no differences observed on day 7 between the DHA- and vehicle-treated animals (Figure $5 \mathrm{~g}$ ).

\section{Discussion}

The present results show that the early neuroinflammatory response mediator NPD1 overcomes cellular damaging events by upregulating the expression of Iduna. We suggest that neural cell homeostasis and function requires induction of critical genes to preserve the integrity of the nervous system. In fact, c-Rel and Birc3 recently were shown to be controlled by NPD1 in a stereoselective-specific manner. ${ }^{17}$ Furthermore, pro- and anti-apoptotic $\mathrm{Bcl}-2$ proteins were shown to be inversely regulated by NPD1 during UOS. ${ }^{11}$ Just as NPD1 also influences the induction of Iduna and the sequestration of the cell death effector PAR, the lipid mediator also targets
PP2A dephosphorylation of Ser-62 of Bcl-xL, which, in turn, binds Bax and prevents activation of the mitochondrial events of apoptosis. ${ }^{18}$ These findings provide evidence that clusters of genes might be transcriptionally coordinated by instructions from NPD1 to resist homeostasis disruptions, where Iduna plays a prominent role.

Thus, protective signaling by NPD1-induced Iduna expression in RPE cells are relevant to sight because these cells sustain photoreceptors integrity and their damage participate in early stages of retinal degenerative diseases. ${ }^{19}$ In fact DHA, the precursor of NPD1, is retained by an intercellular loop between these cells ${ }^{12}$ during photoreceptor outer segment renewal initiated by the phagocytosis of their cell tips. Furthermore, genetic deletion of the adiponectin receptor 1 (AdipoR1), independent of the adiponectin cognate ligand, prevents DHA uptake, blunts NPD1 synthesis and results in ensuing photoreceptor degeneration that resembles certain retinal degenerative diseases. ${ }^{20}$ Moreover, it has recently been demonstrated that mutations on the DHA-retention 
determinant receptor AdipoR1 of photoreceptors leads to certain forms of retinitis pigmentosa ${ }^{21}$ and that a polymorphism of this receptor is involved in age-related macular degeneration in a Finnish population. ${ }^{22}$
Iduna, a PAR-dependent E3 ligase, is involved in protection against neonatal hypoxia-ischemia brain damage. In hypoxia-ischemia encephalopathy, Iduna's downregulation is associated with apoptosis-inducing factor nuclear
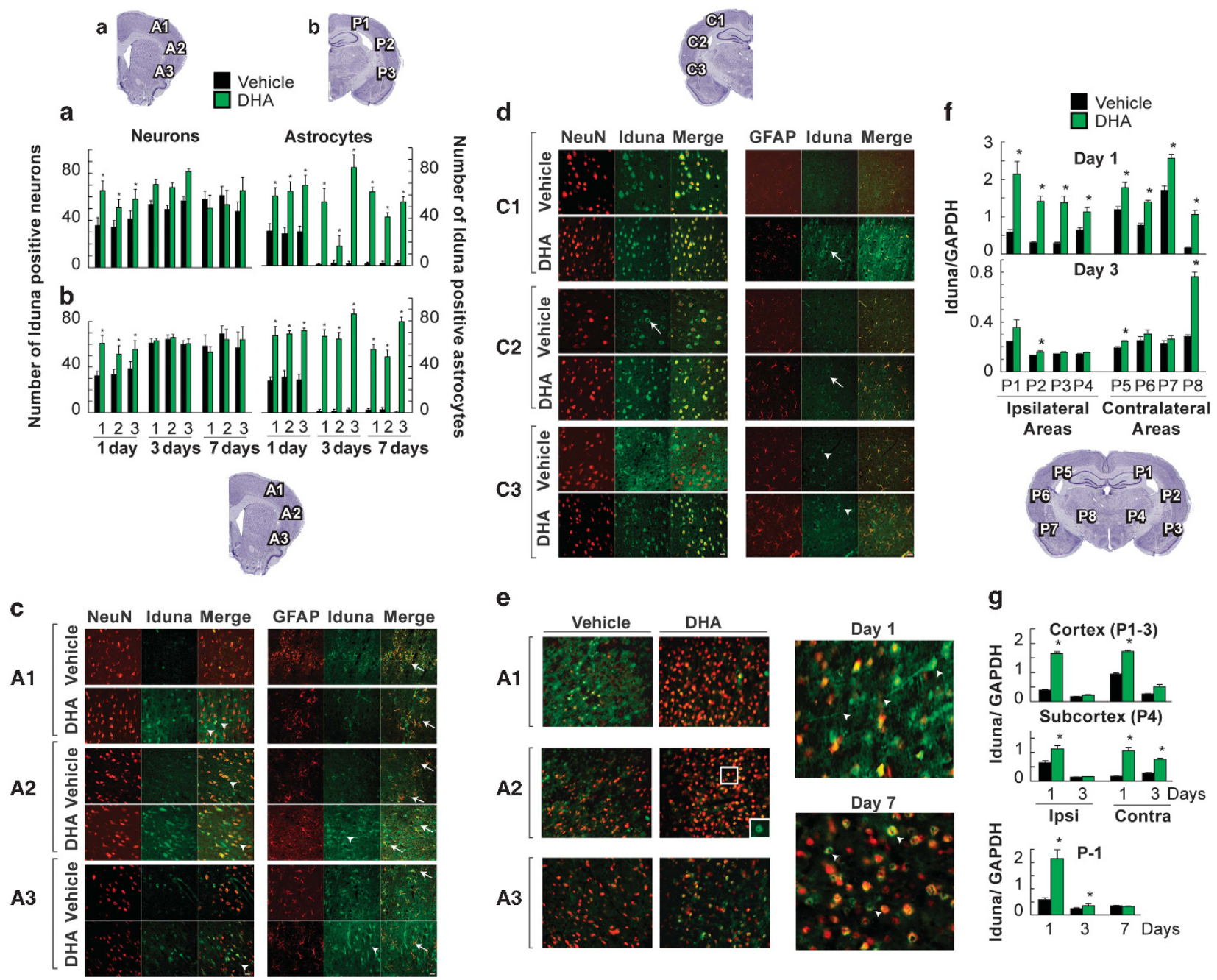

e

g
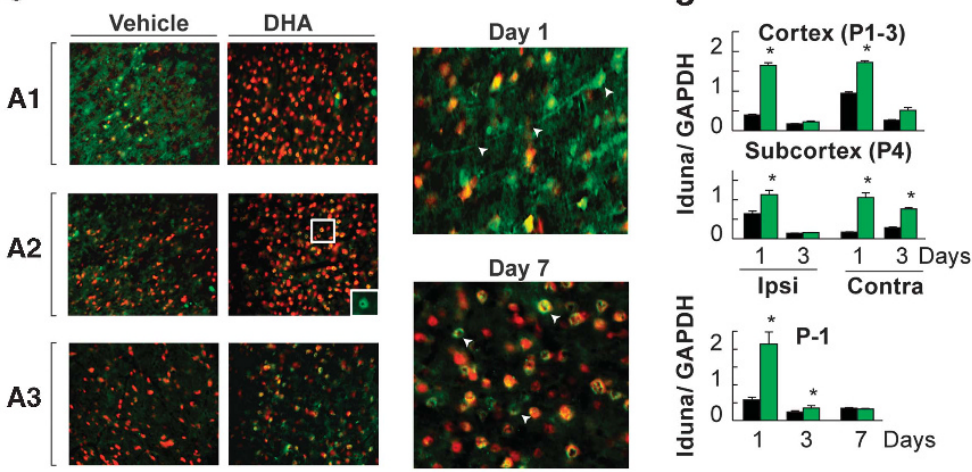

h

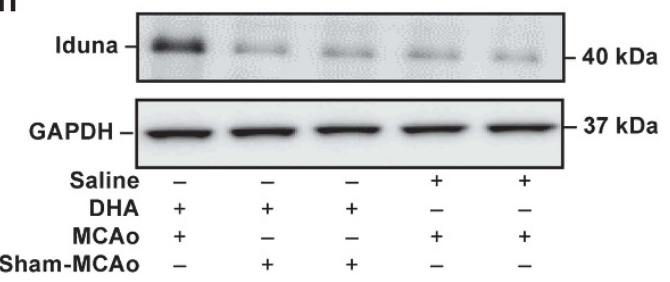

Figure 5 DHA potentiates Iduna expression in neurons and astrocytes. Immunohistochemistry on days 1, 3 and 7 after MCAo. Iduna-positive cells were counted in the cortex at two bregma levels: (a) $+1.2 \mathrm{~mm}(\mathrm{~A} 1, \mathrm{~A} 2, \mathrm{~A} 3)$ and (b) $-2.8 \mathrm{~mm}$ (P1, P2, P3; see diagrams on the top). DHA treatment increased Iduna expression in neurons on day 1 and in astrocytes on days 1, 3 and 7. (c) Iduna expression in the neurons and astrocytes from the ischemic cerebral cortexes (see diagram) of vehicle- and DHA-treated rats on day 1. (d) Iduna expression in the neurons and astrocytes from the contralateral cerebral cortex (see diagram on the top) from vehicle- and DHA-treated rats on day 1. Iduna expression was mostly located in the cytoplasm of the neurons and also expressed in astrocytes. (e) Immunohistochemistry shows a perinuclear Iduna expression compared with vehicletreated animals (picture inside the white box) on days 1 and 7 after DHA treatment. Iduna expression was observed in the nucleus and projections of the neurons on day 1. Contrary to this, perinuclear localization of Iduna occurred on day 7. (f) Western blot: The quantitative data of Iduna expression from ipsi- and contralateral areas on days 1 and 3 , and (g) cortical and subcortical areas (see diagram) on days 1 and 3 and cortex on days 1, 3 and 7. DHA treatment upregulated the expression of Iduna on day 1 and 3 . (g) Immunohistochemical detection of Iduna. (h) Western blot of ipsilateral penumbra on day 1 from MCAo treated with saline, MCAo treated with DHA and sham-MCAo (no MCA occlusion) treated with DHA groups. Data are mean \pm S.E.M. ${ }^{*}$, significantly different from saline $(P<0.05$; repeated measures analysis of variance (ANOVA) followed by Bonferroni tests). Saline $n=7$; DHA $n=6$. Values shown are means \pm S.D. ${ }^{*} P<0.05$, versus saline group (repeated measures ANOVA followed by Bonferroni tests). Iduna (green); NeuN (red), magnification $\times 40$. Neurons (arrows) and astrocytes (arrowheads) 
translocation and neuronal cell death, ${ }^{23}$ and oxidative stressed-DNA damage activates PARP-1. ${ }^{24}$ PARP-1 induces translocation of apoptosis-inducing factor from mitochondria to the nucleus. ${ }^{25}$ Nuclear apoptosis-inducing factor triggers chromatin fragmentation and caspase-independent cell death. ${ }^{26}$ Here we found that inhibitors of PARP alone reduced cell death triggered by oxidative stress in ARPE cells (Figure 2b). When NPD1 was added to the cells treated with PARP inhibitors, a further decrease in cell death was observed with concomitant upregulation of Iduna expression (Figures $2 \mathrm{a}$ and b). These results are in agreement with a partial involvement of PARP-dependent cell death. On the other hand, caspase inhibitors did not induce a complete protection alone, and the addition of NPD1 promoted more survival in cells treated with caspase inhibitors. A recent publication shows that in HT22 cells undergoing UOS, Iduna overexpression does not affect mitochondrial dysfunction to cause cytochrome $c$ release or activation of caspase-3, but instead inhibits activation of PARP-1 and nuclear apoptosis-inducing factor. ${ }^{27}$ Another set of data, however, shows that the effect of Iduna in MCAo rats is PARP-1-independent. ${ }^{7}$ Our conclusion is that more than one cell death type is involved in ARPE-19 undergoing UOS and that NPD1 protects via Iduna induction, at least in part.

It was proposed that both hemispheres act together synergistically to overcome stroke in young rats. ${ }^{28}$ In agreement with this line of thought, we found that Iduna was overexpressed in response to stroke and DHA treatment in both the ipsilateral and contralateral areas. Moreover, contralateral cortical and subcortical areas showed an increase in Iduna abundance at 3 days versus 1 day after MCAo contrary to the ipsilateral counterparts, which involves the penumbra and core of the ischemia-reperfusion lesion. Although Iduna's expression was increased in both hemispheres, subcellular localization differed (Figures $5 c$, d and f). In the ipsilateral area, Iduna was found in the nucleus, where it may be activated by tankyrase, ${ }^{9}$ and has been shown to regulate the turnover of several proteins involved in DNA repair. ${ }^{2}$ On the other hand, cytoplasmic localization of Iduna was found in the contralateral area. The exact function of cytoplasmic Iduna is still under investigation. It was proposed that binding of Iduna with PAR may happen in the cytoplasm and lead to neuroprotection. ${ }^{7}$ As a PAR-dependent E3 ligase, Iduna displays a variety of substrates, and its final effect depends on the specific context in which the enzyme is activated.

In conclusion, the DHA-derived mediator NPD1 enhances Iduna expression under disruptive neurohomeostasis (e.g., UOS or ischemic stroke after MCAo) and thus serves as a key regulator of cell survival, halting neural cell death. The significance of other DHA-derived mediators and pro-cell survival targets of NPD1 remains to be defined. ${ }^{14}$ Thus, further unraveling of the molecular details of DHA-NPD1-Iduna expression signaling may contribute to possible therapeutic interventions for retinal degenerations and ischemic stroke.

\section{Materials and Methods}

Cell cultures, treatments and subcellular fractionation. ARPE-19 cells were grown and maintained in T-75 mM flasks in DMEM F-12 medium containing $10 \%$ FBS and incubated at $37{ }^{\circ} \mathrm{C}$ with a constant supply of $5 \% \mathrm{CO}_{2}$. Similarly, primary human RPE cells were grown in EMEM medium containing $10 \%$
FBS and $5 \%$ fetal calf serum at $37{ }^{\circ} \mathrm{C}$ and $5 \% \mathrm{CO}_{2}$. Cytoplasmic and nuclear fractions were separated by using the Cell Fractionation Kit of Cell Signaling Technology (Danvers, MA, USA) following the protocol supplied by the manufacturers. In some experiments caspases and PARP inhibitors were used. Caspase-1 inhibitor, VX-765; Caspase-3 inhibitor, Z-DEVD (Selleckchem, Houston, TX, USA); Pan caspase inhibitor, Z-VAD (Tocris Bioscience, Bristol, UK); PARP-1 and -2 inhibitor, ABT-888 and a novel PARP inhibitor, AZD2461 (Selleckchem) were added right before induction of UOS.

Transient transfection of ARPE-19 cells by Iduna-GFP constructs. ARPE-19 cells $\left(5 \times 10^{5}\right)$ were transfected with $5 \mu \mathrm{g}$ of human Iduna-GFP constructs (shRNA-Iduna, human YRAA-Iduna) using Fugene-6 according to the manufacturer's protocol (Roach, NJ, USA). In addition, we used Lipofectamine 2000 (Invitrogen, Carlsbad, CA, USA) to transfect cells with siRNAs 1, 2 (InvitrogenThermo cat \# 4392420 inventory \# s37821 and s37822) and scramble (InvitrogenThermo cat\# AM4611) the predesigned RNA duplexes. Transfected cells were incubated $24 \mathrm{~h}$ at $37^{\circ} \mathrm{C}$. They were then serum starved for $8 \mathrm{~h}$ at $37^{\circ} \mathrm{C}$. Serumstarved cells were exposed to UOS $(600 \mu \mathrm{M})$ and treated with NPD1 $(50 \mathrm{nM})$ for $6 \mathrm{~h}$ for western blot analysis and $16 \mathrm{~h}$ for apoptotic cell death assessment at $37^{\circ} \mathrm{C}$.

Western blot analysis. ARPE-19/hRPE cells (grown $72 \mathrm{~h}$ ) in six-well plates were serum starved overnight. UOS was induced by $\mathrm{H}_{2} \mathrm{O}_{2}(600 \mu \mathrm{M})$ plus TNF $\alpha$ $(10 \mathrm{ng} / \mathrm{ml})$, and then cells were challenged with various concentrations $(1 \mathrm{nM}-2 \mu \mathrm{M})$ of NPD1 for 3, 4, 6, 8, 10 and $12 \mathrm{~h}$. Treated cells were harvested, cell lysates were made, protein contents were adjusted and western blot analysis was performed using 20-25 $\mu \mathrm{g}$ protein. Proteins were transferred onto PVDF (Invitrogen) paper and the Iduna protein was detected by either anti-Iduna antibody (RNF146) or clone N201/35 containing anti-Iduna (UC Davis/NIH NeiroMab Facility, Davis, CA, USA).

Immunocytochemistry. Immunocytochemistry was performed on ARPE-19 cells grown on glass slides for $72 \mathrm{~h}$. The cells were serum starved overnight, UOS was induced, and cells treated with 50 and $100 \mathrm{nM}$ NPD1 for $6 \mathrm{~h}$. Then cells were fixed by $4 \%$ paraformaldehyde and probed with an Iduna-specific antibody. To detect the NPD1-mediated expression of Iduna in ARPE-19, paraformaldehyde-fixed treated cells were probed with Iduna-specific antibody, followed by Hoechst $(2 \mu \mathrm{M})$ stain and images were taken by an Axioplan 2 deconvolution microscope and processed with SlideBook 4.2 and 5.0 software (Intelligent Imaging Innovations Inc., Denver, CO, USA).

Immunohistochemistry. Rats were anesthetized and transcardially perfused with $0.9 \%$ saline followed by $4 \%$ paraformaldehyde on days 1,3 and 7 after MCAo. Immunohistochemical procedures were performed on the adjacent sections to identify specific vascular and neuronal elements in the ischemic core and penumbra. Briefly, tissue sections were washed in $1 \times$ PBS and then incubated for $2 \mathrm{~h}$ in a blocking solution (5\% BSA/0.1 Triton X-100 in 1 $\times$ PBS) followed by incubation with the Iduna antibody (1:350; NeuroMab, Davis, CA, USA), NeuN (Chemicon, Temecula, CA, USA) for neurons or GFAP (Cell Signaling, Danvers, MA, USA) to label reactive astrocytes. After incubation, secondary antibodies conjugated with Alexa Flour 488 or 594 were used for $2 \mathrm{~h}$ at room temperature. Sections were mounted on superfros Plus slides and coverslipped with ProLong Gold. Images for counting were obtained using an Axioplan 2 deconvolution microscope and processed with SlideBook 4.2 and 5.0 software (Intelligent Imaging Innovations Inc.). We also used a Zeiss LSM-510 Meta laser confocal microscope with $\times 40$ or $\times 63$ oil to take pictures. Thickness for optical slices of all fluorophores was $11.6 \mu \mathrm{m}$. Image resolution was set to $3.64 \mu \mathrm{m} / \mathrm{pixel}$ and the z-interval was set to $0.91 \mu \mathrm{m}$ to ensure cubic voxel dimensions. Visualization of fluorescent signal was achieved as follows (excitation; emission; pinhole Ø): AlexaFluor 488 (488 nm; 505 LP; 1.00 Airy) AlexaFluor 594 (594 nm; 543 LP; 1.00 Airy). The number of Iduna-positive cells was counted in the three cortical (ischemic penumbra) and one subcortical (ischemic core) areas (ipsi- and contralateral) at the level of the central lesion (bregma $+1.2 \mathrm{~mm}$ and -1.8 ; magnification; $\times 20$ ) using the $\mathrm{NIH}$ Image $J$ program. Data were expressed as numbers of positive cells per highpower microscopic. Quantitative assessment was carried out by an investigator blinded to the experimental groups.

General animal preparation. All experimental protocols were approved by the Institutional Animal Care and Use Committee (IACUC) of the Louisiana State University Health Sciences Center, New Orleans. Male Sprague-Dawley rats (290$320 \mathrm{~g}$; Charles River Lab, Wilmington, MA, USA) were used for in vivo studies. For all surgical procedures, animals were fasted and anesthesia was induced with $3.5 \%$ 
isoflurane and $70 \%$ nitrous oxide and $30 \%$ oxygen. Animals were orotracheally intubated; given atropine for secretions, pancuronium for immobilization; ventilated mechanically on a humidified mixture of $70 \%$ nitrous oxide, $1.0-1.5 \%$ isoflurane and a balance of oxygen. A femoral artery and vein were catheterized for continuous blood pressure monitoring and periodic blood sampling for arterial gases and $\mathrm{pH}$. $\mathrm{PCO}_{2}$ were maintained at $35-40 \mathrm{~mm} \mathrm{Hg}$ and $\mathrm{PO}_{2}$ at $105-120 \mathrm{~mm} \mathrm{Hg}$ by ventilator adjustments. Rectal temperature was measured with a thermistor and maintained with a heating lamp at $37.0-37 \cdot 5^{\circ} \mathrm{C}$. Cranial (temporalis muscle) temperature also was monitored and regulated with a separate warming lamp at $36.2-36.7^{\circ} \mathrm{C}$.

MCA occlusion. The right MCA was occluded for $2 \mathrm{~h}$ by intraluminal filament. ${ }^{12,15}$ Briefly, the right common carotid artery (CCA) was exposed through an incision in the neck. The CCA was isolated from surrounding nerves. The distal external carotid artery (ECA) and pterygopalatine arteries were tied. A 4-cm of 3-0 monofilament nylon suture coated with poly-lysine was introduced via the proximal ECA into the internal carotid artery and MCA. The correct suture position was confirmed by feeling a certain resistance during filament forwarding or by advancing the suture a defined distance $(20-22 \mathrm{~mm})$ from the CCA bifurcation. Then, the animals were allowed to awaken from anesthesia and returned to their cages. The severity of stroke injury was assessed by behavioral examination of each rat at $60 \mathrm{~min}$ after the onset of MCAo. Rats that do not demonstrate high-grade contralateral deficit (score, 10-11) were excluded from further study. After $2 \mathrm{~h}$ of MCA occlusion, the rats were reanesthetized with the same anesthetic combination. Temperature probes were reinserted, and the intraluminal suture was removed carefully. The neck incision was closed with silk sutures, and the animals were allowed to survive for different times (according to the experimental design) with free access to food and water. Rectal and cranial (temporalis muscle) temperatures, plasma glucose, blood pressure and blood gases showed no significant differences among groups. Sham-operated animals underwent all procedures except for MCAo.

A composite neurological battery. This battery consisted of two components: (1) a postural reflex test, designed to examine forelimb and upperbody posture in response to tail-suspension and lateral displacement, regarded as being sensitive to both cortical and striatal lesions; (2) an elicited forelimb placing test, which examines sensorimotor integration by assessing placing reactions to visual, tactile and proprioceptive stimuli. The total neurologic score was graded on a scale from 0 (normal) to 12 (maximal deficit), as we previously described. ${ }^{12,15}$

Treatments. DHA ( $5 \mathrm{mg} / \mathrm{kg}$; Cayman, Ann Arbor, MI, USA; $n=6)$ or vehicle $(0.9 \%$ saline; $n=7$ ) was administered intravenously into the femoral vein at $3 \mathrm{~h}$ after the onset of MCAo. Rats were randomly allocated to experimental groups and treatments.

Magnetic resonance imaging acquisition. High-resolution ex vivo magnetoc resonance imaging (MRI) was performed on $4 \%$ paraformaldehydefixed brains at each time point (days 1, 3 and 7) using an 11.7T Bruker Advance 8.9 $\mathrm{cm}$ horizontal bore instrument equipped with an $89 \mathrm{~mm}$ (ID) receiver coil (Bruker Biospin, Billerica, MA, USA). T2-weighted images (T2WI), diffusion weighted images (DWI), 3D volumes and apparent diffusion coefficient (ADC) maps were collected as we previously described. ${ }^{29}$

MRI image analysis: lesion, core and penumbra volumes. T2 and ADC maps were computed from T2WI and DWI, respectively. We used hierarchical region splitting (HRS) to automatically identify core and penumbra volumes (total lesion = core+penumbra) from T2 relaxation and water mobility (ADC), as we have published previously. ${ }^{29}$ Our penumbral tissue determination by HRS was confirmed by use of PWI/DWI subtractions at each brain level, as we have done previously. ${ }^{29}$ The penumbra will be defined as the difference between the PWI and abnormal ADC (diffusion - perfusion mismatch) (2 STD elevation or reduction compared to normal tissues).

Statistical analysis. Data are presented as mean values \pm S.D. Repeated measure analysis of variance followed by Bonferroni procedures were used for multiple comparisons. Two-tailed Student's t-tests were used for two-group comparisons. Differences at $P<0.05$ were considered statistically significant. Behavioral testing, data acquisition and analysis were performed in a blinded manner.

\section{Conflict of Interest}

The authors declare no conflict of interest.
Acknowledgements. This work was supported by National Eye Institute grant EY005121 (to NGB), National Institute of Neurological Disorders and Stroke grant NS046741 (to NGB), National Institute of General Medicinal Sciences grant GM103340 (to NGB) and in part by an unrestricted departmental grant from the Research to Prevent Blindness, Inc., New York, NY. The shRNA-Iduna and YRAAIduna mouse and human constructs used in these studies were generously provided by Dr. Valina Dawson from Johns Hopkins University School of Medicine.

\section{Author contributions}

All authors contributed to the design and execution of the experiments. LB, LK and SH performed ischemic stroke experiments. PKM and JMC performed retinal pigment epithelial cell cultures. VB performed immunohistochemistry. AO performed magnetic resonance imaging, and $A O, N G B$ and $L B$ analyzed MRI. NGB, LB, JMC and PKM conceived and designed the experiments with input from all co-authors. NGB, LB, JMC and PKM wrote the paper.

1. Zhang J, Li X, Kwansa H, Kim YT, Yi L, Hong G et al. Augmentation of poly(ADP-ribose) polymerase-dependent neuronal cell death by acidosis. J Cereb Blood Flow Metab 2016 (e-pub ahead of print 1 January 2016; doi:10.1177/0271678X16658491).

2. Kang HC, Lee YI, Shin JH, Andrabi SA, Chi Z, Gagné JP et al. Iduna is a poly(ADP-ribose) (PAR)-dependent E3 ubiquitin ligase that regulates DNA damage. Proc Natl Acad Sci USA 2011; 108: 14103-14108.

3. Lee Y, Karuppagounder SS, Shin JH, Lee YI, Ko HS, Swing D et al. Parthanatos mediates AlMP2-activated age-dependent dopaminergic neuronal loss. Nat Neurosci 2013; 16 : 1392-1400.

4. Andrabi SA, Kim NS, Yu SW, Wang H, Koh DW, Sasaki M et al. Poly(ADP-ribose) (PAR) polymer is a death signal. Proc Natl Acad Sci USA 2006; 103: 18308-18313.

5. Krietsch J, Rouleau M, Pic É, Ethier C, Dawson TM, Dawson VL et al. Reprogramming cellular events by poly(ADP-ribose)-binding proteins. Mol Aspects Med 2013; 34: 1066-1087.

6. Zhou ZD, Chan CH, Xiao ZC, Tan EK. Ring finger protein 146/duna is a poly(ADP-ribose) polymer binding and PARsylation dependent E3 ubiquitin ligase. Cell Adh Migr 2011; 5: 463-471.

7. Andrabi SA, Kang HC, Haince JF, Lee Yl, Zhang J, Chi Z et al. Iduna protects the brain from glutamate excitotoxicity and stroke by interfering with poly(ADP-ribose) polymer-induced cell death. Nat Med 2011; 17: 692-699.

8. Gerace E, Landucci E, Scartabelli T, Moroni F, Chiarugi A, Pellegrini-Giampietro DE. Interplay between histone acetylation/deacetylation and poly(ADP-ribosyl)ation in the development of ischemic tolerance in vitro. Neuropharmacology 2015; 92: 125-134.

9. DaRosa PA, Wang Z, Jiang X, Pruneda JN, Cong F, Klevit RE, Xu W. Allosteric activation of the RNF146 ubiquitin ligase by a poly(ADP-ribosyl)ation signal. Nature 2015; 517: 223-226.

10. Bazan NG. Cell survival matters: docosahexaenoic acid signaling, neuroprotection and photoreceptors. Trends Neurosci 2006; 29: 263-271.

11. Mukherjee PK, Marcheselli VL, Serhan CN, Bazan NG. Neuroprotectin D1: a docosahexaenoic acid-derived docosatriene protects human retinal pigment epithelial cells from oxidative stress. Proc Natl Acad Sci USA 2004; 101: 8491-8496.

12. Bazan NG, Molina MF, Gordon WC. Docosahexaenoic acid signalolipidomics in nutrition: significance in aging, neuroinflammation, macular degeneration, Alzheimer's, and other neurodegenerative diseases. Annu Rev Nutr 2011; 31: 321-351.

13. Eady TN, Belayev L, Khoutorova L, Atkins KD, Zhang C, Bazan NG. Docosahexaenoic acid signaling modulates cell survival in experimental ischemic stroke penumbra and initiates long-term repair in young and aged rats. PLOS ONE 2012; 7: e46151.

14. Marcheselli VL, Hong S, Lukiw WJ, Tian XH, Gronert K, Musto A. Novel docosanoids inhibit brain ischemia-reperfusion-mediated leukocyte infiltration and pro-inflammatory gene expression. J Biol Chem 2003; 278: 43807-43817.

15. Bazan NG. Is there a molecular logic that sustains neuronal functional integrity and survival? Lipid signaling is necessary for neuroprotective neuronal transcriptional programs. Mol Neurobiol 2014; 50: 1-5.

16. Belayev L, Khoutorova L, Atkins KD, Eady TN, Hong S, Lu Y, Obenaus A et al. Docosahexaenoic acid therapy of experimental ischemic stroke. Transl Stroke Res 2011; 2: 33-41.

17. Callow MG, Tran H, Phu L, Lau T, Lee J, Sandoval WN et al. Ubiquitin ligase RNF146 regulates tankyrase and Axin to promote Wnt signaling. PLOS ONE 2011; 6: e22595.

18. Calandria JM, Asatryan A, Balaszczuk V, Knott EJ, Jun BK, Mukherjee PK et al. NPD1-mediated stereoselective regulation of BIRC3 expression through CREL is decisive for neural cell survival. Cell Death Differ 2015; 22: 1363-1377.

19. Wright AF, Chakarova CF, Abd El-Aziz MM, Bhattacharya SS. Photoreceptor degeneration: genetic and mechanistic dissection of a complex trait. Nat Rev Genet 2010; 11: 273-284.

20. Rice DS, Calandria JM, Gordon WC, Jun B, Zhou Y, Gelfman CM et al. Adiponectin receptor 1 conserves docosahexaenoic acid and promotes photoreceptor cell survival. Nat Commun 2015; 6: 6228.

21. Xu M, Eblimit A, Wang J, Li J, Wang F, Zhao L et al. ADIPOR1 is mutated in syndromic retinitis pigmentosa. Hum Mutat 2016; 37: 246-249. 
22. Kaarniranta K, Paananen J, Nevalainen T, Sorri I, Seitsonen S, Immonen I et al. Adiponection receptor 1 gene (ADIPOR1) variant is associated with advanced age-related macular degeneration in Finnish population. Neurosci Lett 2012; 513: 233-237.

23. Yang $X$, Cheng J, Gao Y, Ding J, Ni X. Downregulation of Iduna is associated with AIF nuclear translocation in neonatal brain after hypoxia-ischemia. Neuroscience 2017; 346: 74-80.

24. Krietsch J, Caron MC, Gagné JP, Ethier C, Vignard J, Vincent M et al. PARP activation regulates the RNA-binding protein NONO in the DNA damage response to DNA double-strand breaks. Nucleic Acids Res 2012; 40: 10287-10301.

25. Culmsee C, Zhu C, Landshamer S, Becattini B, Wagner E, Pellecchia $M$ et Apoptosis-inducing factor triggered by poly(ADP-ribose) polymerase and Bid mediates neuronal cell death after oxygen-glucose deprivation and focal cerebral ischemia. J Neurosci 2005; 25: 10262-10272.

26. Susin SA, Lorenzo HK, Zamzami N, Marzo I, Snow BE, Brothers GM et al. Molecular characterization of mitochondrial apoptosis-inducing factor. Nature 1999; 397 : 441-446.

27. Xu H, Luo P, Zhao Y, Zhao M, Yang Y, Chen T et al. Iduna protects HT22 cells from hydrogen peroxide-induced oxidative stress through interfering poly(ADP-ribose) polymerase-1induced cell death (parthanatos). Cell Signal 2013; 25: 1018-1026.
28. Buga AM, Sascau M, Pisoschi C, Herndon JG, Kessler C, Popa-Wagner A. The genomic response of the ipsilateral and contralateral cortex to stroke in aged rats. $J$ Cell Mol Med 2008; 12: 2731-2753.

29. Obenaus A, Dilmac N, Tone B, Tian HR, Hartman R, Digicaylioglu M et al. Long-term magnetic resonance imaging of stem cells in neonatal ischemic injury. Ann Neurol 2011; 69: 282-291.

(c) (i) This work is licensed under a Creative Commons Attribution 4.0 International License. The images or other third party material in this article are included in the article's Creative Commons license, unless indicated otherwise in the credit line; if the material is not included under the Creative Commons license, users will need to obtain permission from the license holder to reproduce the material. To view a copy of this license, visit http:// creativecommons.org/licenses/by/4.0/

(C) The Author(s) 2017 